\title{
Recent developments of SOLEROO: Australia's first high energy radioactive Ion Beam capability
}

\author{
I. P. Carter ${ }^{1, a}$, M. Dasgupta ${ }^{1}$, D. J. Hinde ${ }^{1}$, D. H. Luong ${ }^{1}$, E. Williams ${ }^{1}$, K. Ramachandran ${ }^{1, b}$, K. J. Cook ${ }^{1}$, A. G. \\ Muirhead $^{1}$, S. Marshall ${ }^{1}$, and T. Tunningley ${ }^{1}$ \\ ${ }^{1}$ Department of Nuclear Physics, Research School of Physics and Engineering, Australian National University, ACT 0200, \\ Australia
}

\begin{abstract}
The first measurements of the Australian National University's new radioactive ion beam capability were carried out using elastic scattering of a ${ }^{8} \mathrm{Li}$ radioactive beam from a ${ }^{197} \mathrm{Au}$ target. The purpose of this experiment was to test the radioactive ion beam capability as a complete system, which uses a pair of twin position-sensitive parallel plate avalanche counters as tracking detectors along with a highly pixelated double sided Si detector array. The tracking detector system allows us to have extremely high purity secondary radioactive ion beams by electronically tagging the reaction products of interest, thus allowing complete separation from the unwanted contaminant beam species of similar mass and charge. Here, some recent developments and characteristics of this system are presented.
\end{abstract}

\section{Introduction}

The production of radioactive ion beams (RIBs) around the world has opened up the possibility of exploring a new range of physics questions. The Australian National University (ANU) RIB capability (know as SOLEROO) is based on a super-conducting solenoidal separator. This capability, which is almost ready for experiments, can produce light radioactive nuclei such as $\left({ }^{6} \mathrm{He},{ }^{8} \mathrm{Li},{ }^{10} \mathrm{Be},{ }^{12} \mathrm{~B}\right)$ $[1,2]$. These RIBs are produced by in-flight transfer reactions with the primary target in the production chamber. The transfer products enter a magnetic field region produced by a single solenoid with an angular acceptance of $2^{\circ}-6^{\circ}$ and the desired RIB species are deflected by the high axial magnetic field and are focused onto the secondary target located on the beam axis outside the solenoid. A single solenoidal separator may achieve purities up to $60 \%$. At other facilities two solenoids have been used in tandem to further purify the RIBs e.g. at TwinSol [3] and RIBRAS [4]. At the ANU, further purification of the beam is achieved using a pair of tracking detectors. Each ion exiting the solenoid prior to reaching the secondary target passes through the tracking detectors, where they are electronically tagged on an event-by-event basis. This allows reconstruction of ion trajectories to aid in rejection of contaminant species. It was found that a tracking system based on Parallel Plate Avalanche Counters (PPACs) had suitable properties to work under the high-count rate environment to which they would be subjected. This proceed-

\footnotetext{
a e-mail: ian.carter@anu.edu.au

bermanent address: Nuclear Physics Division, Bhabha Atomic Research Centre, Trombay, Mumbai, India
}

ings aims to present an overview of recent developments at the ANU radioactive beam capability.

\section{RIB tracking system}

The essential idea of using the tracking detectors is to tag and purify the RIB species from information on: (1) the ion trajectories; (2) the energy loss of the ions passing through the detectors and (3) the time of flight of ions between the detectors. In order to be effective the detectors must satisfy the following conditions:

(i) Be transparent to the incident ions with minimum beam angular straggling;

(ii) High count rate capability up to $10^{6}$ counts/s;

(iii) High efficiency;

(iv) Excellent timing properties;

(v) Position sensitivity of $\leq 1 \mathrm{~mm}$;

(vi) Radiation hard.

A PPAC is a gas detector that operates in the proportional gas-amplification region [1, 5-10]. PPACs in principle can meet the above criteria; the challenge is to realise all these capabilities. Separation of the RIB from the impurities of similar mass and flight time is achieved through utilising the fast timing properties and the different energy deposited in these detectors by different ion species. The time of flight (TOF) of the incoming ions between the two detectors is proportional to $\sqrt{\frac{m}{E}}$, where $m$ is the mass and $E$ is the energy of the incoming ion. The energy lost by 
these ions in the detectors is proportional to $\frac{m Z^{2}}{E}$, where $Z$ is the mean ionic charge in the PPAC gas. These combined quantities allows the tracking system to electronically reject the large flux of contaminant species and select the RIBs of interest [5].

Another important function of the system is the ability to track the trajectories of the ions passing through the detectors onto a secondary target placed downstream from these detectors. This is vital because the RIB beam spot is not as small compared to that of a stable beam. Therefore, knowledge of the interaction point of each ion colliding with the secondary target is important. This is achieved through the position sensitivity in the tracking detector system.

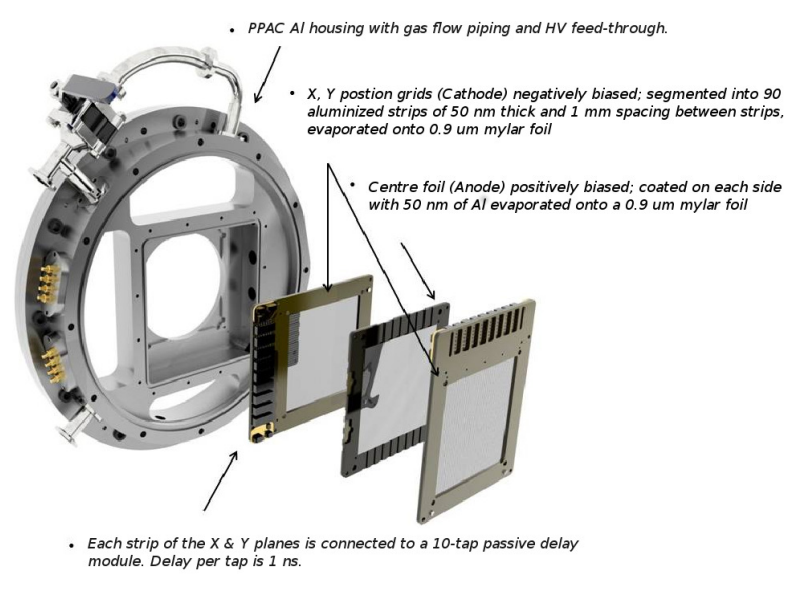

Figure 1. Expanded view of one of the PPACs developed at the ANU for tagging RIBs.

Figure 1 shows an expanded view of the PPAC developed at ANU. It consists of a square active area of three $90 \times 90 \mathrm{~mm}$ metallized foils that are mounted parallel to each other. The position foils are segmented into metallized strips and rotated $90^{\circ}$ from each other, allowing the detector to be position sensitive. Described in detail in [5], the position response of this system was shown to be linear, with a position resolution $\leq 1 \mathrm{~mm}$ FWHM.

\section{PPAC Characteristics}

This section will present an overview of the tracking systems performance in terms to the prerequisites listed in section 2 .

\subsection{Transparent to incident ions}

Gas detectors are less sensitive to radiation damage and their effective thickness can be changed easily by changing the gas pressure within the volume. Therefore, the PPACs can be tuned to be almost transparent to the incident ions. Furthermore, it is desirable that the secondary RIBs do not lose a significant amount of energy in these detectors causing angular straggling and degradation of the secondary beam profile. These effects were found to be negligible for a working gas pressure of 10 Torr (the nominal gas pressure used inside the detectors) [1].

\subsection{High count rate capability}

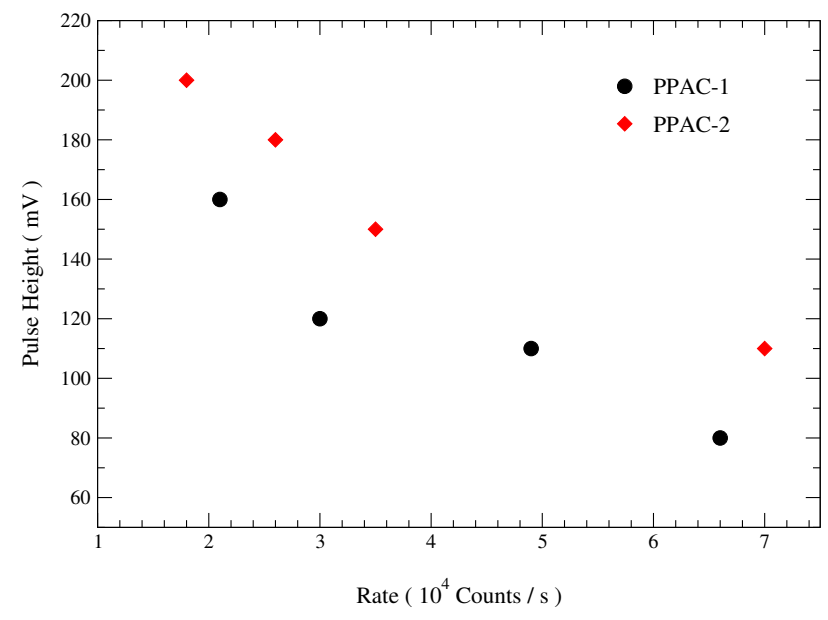

Figure 2. Measured pulse height with increasing count rates.

The ability of the tracking system to handle high count rates on the order of $10^{6}$ particles per second for long periods of time is vital, so that the tracking system does not limit the RIB intensity. The rate handling ability was tested and is shown in Figure 2. For a fixed total bias voltage of $730 \mathrm{~V}$ on the detectors, as the particles rate on the detectors was increased, the measured pulse height for events passing through the PPACs reduced. This is due to space charge effects: as electrons are liberated in the gas, they form a cloud of charge, reducing the electric field strength. This limits the signal amplification in the gas. To compensate for the lower pulse height, which results in a lower detection efficiency with increasing rate, the detector bias has to be increased, increasing the risk of unstable operation where breakdown can occur.

The position detection efficiency of ${ }^{6} \mathrm{He}$ was measured at different bias voltages as a function of count rate on PPAC-2, the detector placed furthest downstream. The results are shown in Figure 3 where the highest rate on PPAC- 2 was measured at $>7.0 \times 10^{5}$ counts/s at a bias voltage of $900 \mathrm{~V}$ using a filling gas of octafluropropane. Figure 3 indicates these detectors are easily handling rates greater that $10^{5}$ counts/s, with the overall efficiency dropping off at larger count rates as expected.

\subsection{Detection efficiency}

Figure 3 shows that as the bias voltage is increased, the detection efficiency increases, with the efficiency approaching $97 \%$ at $930 \mathrm{~V}$. Although there aren't many data points for the measurements at $930 \mathrm{~V}$ and $950 \mathrm{~V}$, one would expect the trend to be the same, as seen at $900 \mathrm{~V}$, a gradual decrease in efficiency as the count rate is increased. The sudden drop in efficiency at a bias voltage of $980 \mathrm{~V}$ at $\approx$ $6.0 \times 10^{5}$ counts/s is interesting. PPAC- 1 , closest to the exit 
of the solenoid, had actually broken down at this bias and rate. Therefore it would not be surprising that the sudden drop in efficiency in PPAC-2 is a signature that this detector was also close to breaking down. Operating in an unstable region, it was likely undergoing corona discharges between the point of primary ionization in the gas and the electrodes. Therefore, the ideal working bias for this filling gas in the detector volume is $\approx 930 \mathrm{~V}$, a bias which gives high efficiency for the detection of ${ }^{6} \mathrm{He}$ ions (the lowest mass RIB to be studied) and operation at a bias $\approx 50 \mathrm{~V}$ less than the breakdown voltage of $980 \mathrm{~V}$. In order to comfortably carry out experiments with this beam species, it is vital that the tracking system can operate at a working bias that gives high efficiency for its detection at rates $>10^{5}$ counts/s, as has been demonstrated.

As seen in Figure 4, the detection efficiency of ${ }^{6} \mathrm{He}$ and the other reaction products as a function of applied bias voltage for a field optimised for the transmission of ${ }^{6} \mathrm{He}$ at a rate of $\approx 4 \times 10^{4}$ counts/s. The efficiency of ${ }^{6} \mathrm{He}$ is $\approx 97 \%$ at $930 \mathrm{~V}$, falling off quickly at lower bias voltages. The elastic ${ }^{7} \mathrm{Li}$ ions were detected with an efficiency close to $100 \%$ at every measured bias. This suggests that the detection efficiency for any ion with $\mathrm{Z} \geq 3$ e.g. RIBs of ${ }^{8} \mathrm{Li}$ will not pose a problem.

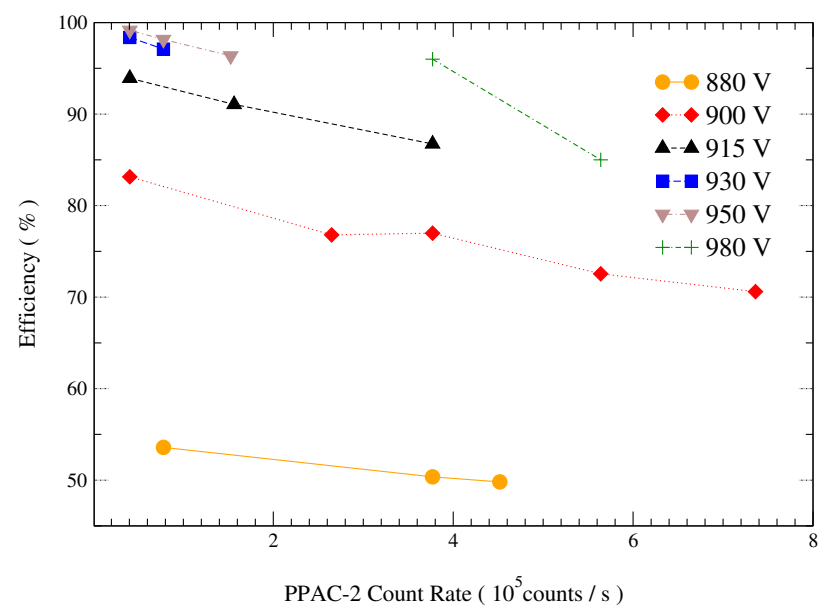

Figure 3. Detection efficiency of ${ }^{6} \mathrm{He}$ as a function of count rate as the bias voltage is increased, for octafluoropropane detector gas at 10 torr pressure.

\subsection{Timing properties}

The timing and energy resolution of the PPACs were measured as a function of applied bias, and using two types of filling gas: propane $\left(\mathrm{C}_{3} \mathrm{H}_{8}\right)$ and octafluoropropane $\left(\mathrm{C}_{3} \mathrm{~F}_{8}\right)$. Initially, $\mathrm{a}^{241} \mathrm{Am}$ source emitting $5.5 \mathrm{MeV} \alpha$-particles was placed in front of the PPACs and used to measure the detector's timing and energy response. Then higher energy secondary beams of ${ }^{6} \mathrm{He}$ and ${ }^{8} \mathrm{Li}$ produced in our RIB system were used to measure the timing and energy response. Table 1 summarises the results of these measurements. What is clear from this table is the good timing properties of the PPACs, having a resolution in the sub-nanosecond range. For the reaction groups listed in Table 1, the time

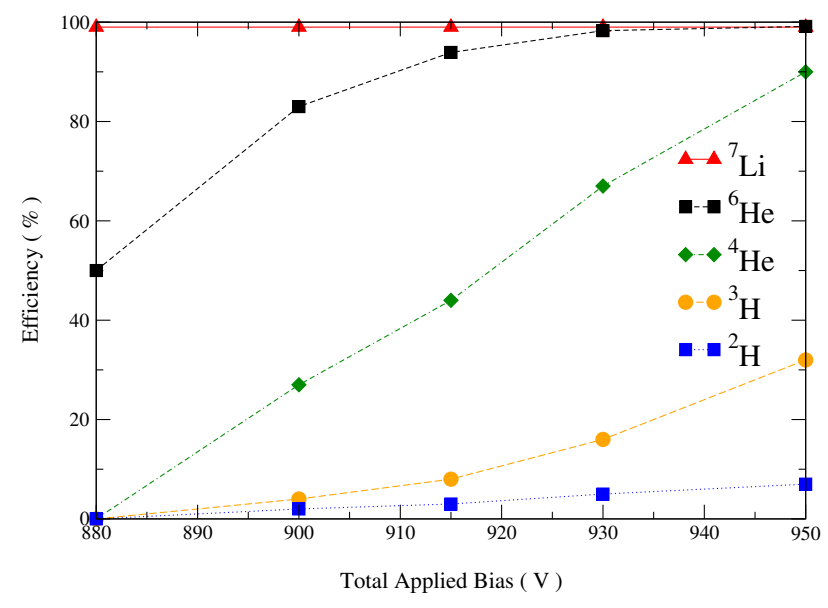

Figure 4. Efficiency of ${ }^{2} \mathrm{H},{ }^{3} \mathrm{H},{ }^{4} \mathrm{He},{ }^{6} \mathrm{He}$ and ${ }^{7} \mathrm{Li}$ as a function of applied bias voltage for a solenoidal magnetic field optimised for the transmission of ${ }^{6} \mathrm{He}$.

resolution was obtained from the full width at half maximum (FWHM) of the measured time of flight distribution between the two PPACs, divided by $\sqrt{2}$, assuming both detectors timing response were the same. This was achieved after gating the reaction products on a Silicon $\Delta E$-E detector that can be placed in the position of the secondary target. This allowed for a clean separation of ion species when projecting back onto the PPACs, to better examine the coincident timing events. The energy response from the PPACs was obtained in the similar way, but is displayed as a percentage of the ratio $\left(\frac{F W H M}{\text { MeanEnergy }}\right)$ as no absolute calibration of energy loss in the PPACs was obtained.

From the ${ }^{241}$ Am source measurements shown in Table 1 , it was noticed as the bias voltage was increased the timing response improved. The energy response showed signs of a slight decrease in resolution, by about 6-7\%, possibly because more electrons were collected across the gap between the electrodes. These electrons can interact with the filling gas in secondary ionization processes and cause the energy response to decrease as the bias is increased. The bias voltage was increased to a maximum value of $800 \mathrm{~V}$ for propane and $930 \mathrm{~V}$ for octafluoropropane. The characteristics of the detectors will vary slightly with gas because the primary ionization in the gas will depend on what gas is used. The bias voltage was set for both gases so that pulse heights from the detectors were approximately the same. Then, to ensure that operation was in a stable region, the bias was reduced by $50 \mathrm{~V}$ from their breakdown voltage. For ${ }^{6} \mathrm{He}$ measured using octafluoropropane, both the time and energy responses are better compared to the measurement with propane, presumably due to more primary ionization in the gas.

\subsection{Position sensitivity}

Position sensitivity was described in a previous proceedings [5]. The position response was measured by placing position mask placed in front of the PPACs and illuminat- 
Table 1. PPAC characteristics.

\begin{tabular}{cccccccc}
\hline \hline RIB & $\begin{array}{c}\text { Energy } \\
\mathrm{MeV}\end{array}$ & $\begin{array}{c}{ }^{9} \text { Be Production Target } \\
\frac{m g}{c m^{3}}\end{array}$ & Filling Gas & $\begin{array}{c}\text { Gas Pressure } \\
\text { Torr }\end{array}$ & $\begin{array}{c}\text { Total Bias Applied } \\
\text { V }\end{array}$ & $\begin{array}{c}\text { Time Resolution } \\
\text { FWHM }(\mathrm{ps})\end{array}$ & $\begin{array}{c}\text { Energy Resolution } \\
\%\end{array}$ \\
\hline${ }^{4} \mathrm{He}$ & 5.50 & N/A & $\mathrm{C}_{3} \mathrm{~F}_{8}$ & 10 & 900 & 547 & 26.35 \\
${ }^{4} \mathrm{He}$ & 5.50 & N/A & $\mathrm{C}_{3} \mathrm{~F}_{8}$ & 10 & 920 & 497 & 29.23 \\
${ }^{4} \mathrm{He}$ & 5.50 & $\mathrm{~N} / \mathrm{A}$ & $\mathrm{C}_{3} \mathrm{~F}_{8}$ & 10 & 950 & 447 & 31.67 \\
${ }^{4} \mathrm{He}$ & 5.50 & $\mathrm{~N} / \mathrm{A}$ & $\mathrm{C}_{3} \mathrm{~F}_{8}$ & 10 & 980 & 399 & 30.85 \\
${ }^{8} \mathrm{Li}$ & $29.87-30.13$ & 2.79 & $\mathrm{C}_{3} \mathrm{H}_{8}$ & 10 & 750 & 588 & 53.63 \\
${ }^{6} \mathrm{He}$ & $25.95-26.18$ & 2.79 & $\mathrm{C}_{3} \mathrm{H}_{8}$ & 10 & 800 & 610 & 69.53 \\
${ }^{6} \mathrm{He}$ & $25.95-26.18$ & 2.79 & $\mathrm{C}_{3} \mathrm{~F}_{8}$ & 10 & 930 & 617 & 44.94 \\
\hline
\end{tabular}

ing them with a ${ }^{241} \mathrm{Am} \alpha$-source. The position resolution was found to be better than $1 \mathrm{~mm}$ FWHM.

\subsection{Radiation hardness (stability of operation and improvements)}

In order to comfortably carry out experiments with low mass RIBs it is crucial that the detectors are able to operate for long periods of time (weeks). To ensure that they could operate for a long duration at such a high count rate, it was decided to improve the system by making our detectors smaller. Making the detectors physically smaller reduces the capacitance of the electrodes according to:

$$
C=\frac{Q}{V}=\epsilon_{o} \frac{A}{d}
$$

Where $C$ is the capacitance between the electrodes, $Q$ is the charge, $\epsilon_{o}$ is the permittivity of free space, $A$ is the surface area of the electrode and $d$ is the distance between the electrodes. It was found from recent experiments with ${ }^{8} \mathrm{Li}$ beams that the beam profile on the first PPAC located downstream from the solenoid was $<30 \mathrm{~mm}$ in diameter. As this detector dictates the acceptance of beam being brought to a focus on the secondary target, the active area of the PPAC could be made smaller, from $90 \mathrm{~mm}^{2}$ to 35.5 $\mathrm{mm}^{2}$, resulting in 6.4 times less capacitance on the detectors and much larger signals for a given bias voltage.

Recently tests of the stability of operation for these smaller PPACs was carried out using ${ }^{241} \mathrm{Am} \alpha$-source, placed directly in front of the PPACs with rates $\approx 2 \times 10^{4}$ counts/s for 60 hours. There was no sign of degradation in the cathode or the anode of both detectors. In both the older and newer versions of the detector, the cathode and anode were coated with $\mathrm{Al}$ which was found to have a higher radiation hardness than the typically used Au [6]. Kumagi et al. [6], previously performed a similar test where they measured the stability of operation for a PPAC with a cathode coated with Au or Al. They applied count rates of $\approx 2.5 \times 10^{4}$ counts/s using a $61.9 \mathrm{~A} \mathrm{MeV}{ }^{8} \mathrm{Li}$ beam for about 7 hours. After the measurements, they compared the damage to the cathode and noted a clear difference in the size of discharge traces under the microscope. Damaged traces on the Au cathode were bigger than those on the Al. No signs of damage were seen in our tests.

\section{The complete RIB capability}

The first radioactive ion beam experiment has recently been carried out at the ANU, where the RIB capability
SOLEROO [1, 2, 5] was tested with the tracking detector system integrated with a silicon detector array [BALIN], developed for breakup measurements [11-20]. In order to test the integrated system as a whole, elastic scattering measurements were carried out using a tagged ${ }^{8} \mathrm{Li}$ secondary beam incident on a ${ }^{197} \mathrm{Au}$ target. The beam energy was below the barrier where Rutherford scattering was expected; the results of the measurement are shown in Figure 5. The energy of the ${ }^{8} \mathrm{Li}$ events were measured as a function of $\theta$, as shown in Figure 5. This was a test experiment and in comparison with a real RIB experiment, it was a short run where we had a primary beam intensity of $\approx 10 \%$ of the beam intensity planned for future RIB experiments. The events in Figure 5 appearing backward at around $90^{\circ}$ at low energy are possibly from the secondary beam hitting the target frame. It was found that the production rates for ${ }^{6} \mathrm{He}$ were $5.0 \times 10^{4} \mathrm{cts} / \mathrm{sec} / \mathrm{mg} / \mu \mathrm{eA}$ and for ${ }^{8} \mathrm{Li} 2.86 \times 10^{5} \mathrm{cts} / \mathrm{sec} / \mathrm{mg} / \mu \mathrm{eA}$. The purity of the tagged RIB ${ }^{6} \mathrm{He}$ using the PPACs were found to be $>92 \%$. On the other-hand, for ${ }^{8} \mathrm{Li}$, using the TOF between the two detectors one could only achieve purities up to $85 \%$, with a large amount of impurities coming from energy degraded ${ }^{7} \mathrm{Li}^{3+}$ with the same flight time. In order to increase the timing separation, the timing signal was measured from one PPAC detector relative to a pulsed beam. By doing this, the purity of ${ }^{8} \mathrm{Li}$ increased to $>95 \%$ of the tagged secondary beam. However, as it was needed to bunch and chop the primary beam, the primary beam intensity was reduced by about $75 \%$.

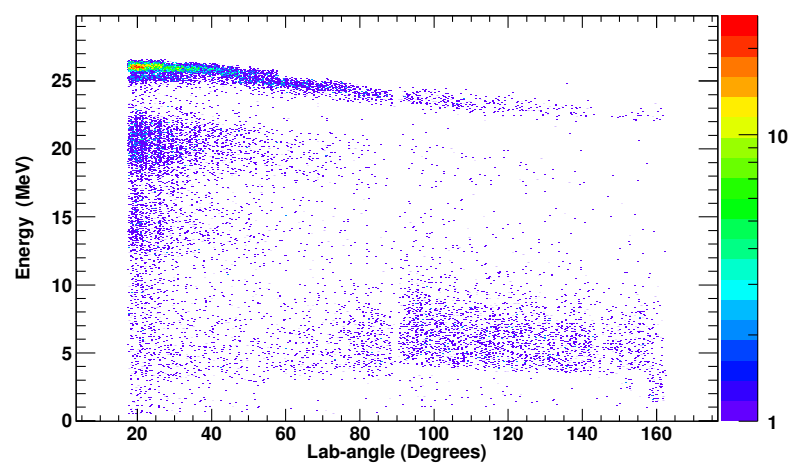

Figure 5. Measured energy as a function of $\theta$ for elastic scattering of a tagged ${ }^{8} \mathrm{Li}$ secondary beam from a $1 \mathrm{mg} / \mathrm{cm}^{3}{ }^{197} \mathrm{Au}$ target. 


\section{Conclusion}

A tracking system has been designed for use in the RIB separator which allows separation of RIB reaction products from contaminant beam species of similar mass and charge. The overall detection efficiency is high and the timing characteristics are in the sub-nanosecond range. The detectors have been tested with rates over $10^{5}$ counts/s. The long-term stability of the detectors has been addressed by reducing the active area of the electrodes from $90 \mathrm{~mm}^{2}$ to $35 \mathrm{~mm}^{2}$, reducing the capacitance. The detectors were tested with an $\alpha$-source with rates of $10^{4}$ counts/s for 60 hours and showed no sign of degradation in performance or any physical defects. Although a test with a higher count rate for a long duration of time would be desirable, this preliminary test on the long-term stability suggests the stability of operation for long periods of time with high count rates will not be an issue.

The RIB capability as a complete system has been tested for the very first time. Everything is working satisfactorily; achieving high purity RIBs by tagging with the tracking detector system. Experiments measuring elastic scattering and breakup reaction are being planned with secondary RIBs of ${ }^{6} \mathrm{He}$ and ${ }^{8} \mathrm{Li}$ in the near future.

\section{Acknowledgements}

The authors would like to acknowledge R. Rafiei, A.J. Horseley and A. B. Harding for their excellent experimental and technical contribution towards the development of the first energetic radioactive ion beam capability in Australia. They would also like to acknowledge the contributions made by S. Kalkal, E. Prasad and D.Y. Jeung during the recent test experiment with our first radioactive beam of ${ }^{8} \mathrm{Li}$. This work was supported by the ARC Discovery Grant DP0879679, DP110102879, DP130101569 and FL110100098.

\section{References}

[1] R. Rafiei, D.J. Hinde, M. Dasgupta, D.C. Weisser, A.G. Muirhead, A.B. Harding, A.K. Cooper, H.J. Wallace, N.R. Lobanov, A. Wakhle et al., Nucl. Instruments Methods Phys. Res. Sect. A Accel. Spectrometers, Detect. Assoc. Equip. 631, 12 (2011)

[2] A. Horsley, D. Hinde, M. Dasgupta, R. Rafiei, A. Wakhle, M. Evers, D. Luong, R. du Rietz, Nucl. Instruments Methods Phys. Res. Sect. A Accel. Spectrometers, Detect. Assoc. Equip. 646, 174 (2011)

[3] M.Y. Lee, F.D. Becchetti, J.M. Holmes, T.W. O’Donnell, M.A. Ratajczak, D.A. Roberts, J.A. Zimmerman, J.J. Kolata, L.O. Lamm, J. von Schwarzenberg et al., AIP Conference Proceedings 392 (1997)

[4] R. Lichtenthäler, a. Lépine-Szily, V. Guimar aes, C. Perego, V. Placco, O. Camargo jr., R. Denke, P.N. de Faria, E.a. Benjamim, N. Added et al., Eur. Phys. J. A 25, 733 (2005)

[5] I.P. Carter, K. Ramachandran, M. Dasgupta, D.J. Hinde, R. Rafiei, D.H. Luong, E. Williams, K.J. Cook, S. McNeil, D.C. Rafferty et al., EPJ Web of Conferences 63, 02022 (2013)

[6] H. Kumagai, A. Ozawa, N. Fukuda, K. Sümmerer, I. Tanihata, Nucl. Instruments Methods Phys. Res. Sect. A Accel. Spectrometers, Detect. Assoc. Equip. 470, 562 (2001)

[7] D. Swan, J. Yurkon, D.J. Morrissey, Nucl. Instruments Methods Phys. Res. Sect. A Accel. Spectrometers, Detect. Assoc. Equip. 348, 314 (1994)

[8] A. Breskin, Nucl. Instruments Methods Phys. Res. 196, 11 (1982)

[9] S. Ottini-Hustache, C. Mazur, F. Auger, A. Musumarra, N. Alamanos, B. Cahan, A. Gillibert, A. Lagoyannis, O. Maillard, E. Pollacco et al., Nucl. Instruments Methods Phys. Res. Sect. A Accel. Spectrometers, Detect. Assoc. Equip. 431, 476 (1999)

[10] J. Cub, C. Gund, D. Pansegrau, G. Schrieder, H. Stelzer, Nucl. Instruments Methods Phys. Res. Sect. A Accel. Spectrometers, Detect. Assoc. Equip. 453, 522 (2000)

[11] R. Rafiei, R. du Rietz, D.H. Luong, D.J. Hinde, M. Dasgupta, M. Evers, A. Diaz-Torres, Phys. Rev. C 81, 24601 (2010)

[12] M. Dasgupta, D. Hinde, R.D. Butt, R. Anjos, A. Berriman, N. Carlin, P. Gomes, C. Morton, J. Newton, A.S. De Toledo et al., Physical review letters 82, 1395 (1999)

[13] M. Dasgupta, D. Hinde, K. Hagino, S. Moraes, P. Gomes, R. Anjos, R. Butt, A. Berriman, N. Carlin, C. Morton et al., Phys. Rev. C 66, 041602 (2002)

[14] M. Dasgupta, P. Gomes, D. Hinde, S. Moraes, R. Anjos, A. Berriman, R. Butt, N. Carlin, J. Lubian, C. Morton et al., Phys. Rev. C 70, 024606 (2004)

[15] D.J. Hinde, M. Dasgupta, B.R. Fulton, C.R. Morton, R.J. Wooliscroft, A.C. Berriman, K. Hagino, Phys. Rev. Lett. 89, 272701 (2002)

[16] D.J. Hinde and M. Dasgupta, Nature 431, 748 (2004)

[17] K. Cook, D. Luong, E. Williams, EPJ Web of Conferences 35, 05004 (2012)

[18] K. Cook, D. Luong, E. Williams, I. Carter, M. Dasgupta, D. Hinde, K. Ramachandran, EPJ Web Conf. 63, 03011 (2013)

[19] D. Luong, M. Dasgupta, D. Hinde, R.D. Rietz, R. Rafiei, C. Lin, M. Evers, A. Diaz-Torres, Phys. Lett. B 695, 105 (2011)

[20] D.H. Luong, M. Dasgupta, D.J. Hinde, R. du Rietz, R. Rafiei, C.J. Lin, M. Evers, A. Diaz-Torres, Phys. Rev. C 88, 34609 (2013) 